\title{
Sensitivity of the Product System of Set-Valued Non-Autonomous Discrete Dynamical Systems
}

\author{
Yongxi Jiang1,2, Tianxiu Lu${ }^{1 *}$, Jingmin $\mathrm{Pi}^{1}{ }^{1}$, Xiaofang Yang1,2 \\ ${ }^{1}$ College of Mathematics and Statistics, Sichuan University of Science and Engineering, Zigong, China \\ ${ }^{2}$ Sichuan Education of Sichuan Province for Enterprise Informationalization and Internet of Things, Zigong, China \\ Email: jyx970817@163.com, ^lubeeltx@163.com,pi1011225770@126.com,yxf_suse@163.com
}

How to cite this paper: Jiang, Y.X., Lu, T.X., Pi, J.M. and Yang, X.F. (2021) Sensitivity of the Product System of Set-Valued Non-Autonomous Discrete Dynamical Systems. Journal of Applied Mathematics and Physics, 9, 2706-2716.

https://doi.org/10.4236/jamp.2021.911174

Received: October 1, 2021

Accepted: November 7, 2021

Published: November 10, 2021

Copyright $\odot 2021$ by author(s) and Scientific Research Publishing Inc. This work is licensed under the Creative Commons Attribution International License (CC BY 4.0).

http://creativecommons.org/licenses/by/4.0/

\section{Abstract}

This paper is concerned with the sensitivity of set-valued discrete systems. Firstly, this paper obtained the equivalence between $\left(\mathcal{K}(X), \bar{f}_{1, \infty}\right)$ or $\left(\mathcal{K}(Y), \bar{g}_{1, \infty}\right)$ and the product system $\left(\mathcal{K}(X \times Y), \overline{f_{1, \infty} \times g_{1, \infty}}\right)$ in sensitivity, infinite sensitivity, $\mathcal{F}$-sensitivity, $\left(\mathcal{F}_{1}, \mathcal{F}_{2}\right)$-sensitivity. Then, the relation between $\left(X, f_{1, \infty}\right)$ or $\left(Y, g_{1, \infty}\right)$ and $\left(\mathcal{K}(X \times Y), \overline{f_{1, \infty} \times g_{1, \infty}}\right)$ in ergodic sensitivity is obtained. Where $\left(\mathcal{K}(X), \bar{f}_{1, \infty}\right)$ is the set-valued dynamical system induced by a non-autonomous discrete dynamical system $\left(X, f_{1, \infty}\right)$.

\section{Keywords}

Sensitivity, Set-Valued System, Product Mapping, Non-Autonomous Systems

\section{Introduction}

Since the beginning of the 21 st century, the problem of chaos in set-valued discrete systems has been discussed warmly. In 2003, Román-Flores [1] studied the interaction of transitivity between $(X, f)$ and its induced system $(\mathcal{K}(X), \bar{f})$. Following his work, many scholars studied the chaotic properties of set-valued discrete systems. For example, transitivity, mixing, Kato's chaos (see [2]-[9] and others). About sensitivity, Liu, Shi, and Liao [10] proved that if $f$ is a surjective, continuous interval map, then $\bar{f}$ is sensitive if and only if $f$ is sensitive. And in 2013, they gave an example to show that Li-Yorke sensitivity of $f$ does not necessarily imply Li-Yorke sensitivity of $\bar{f}$ ([11]). In 2010, Sharma and Nagar [12] showed the relations between the various forms of sensitivity of the systems $(X, f)$ and $(\mathcal{K}(X), \bar{f})$ and proved that all forms of sensitivity of 
$(\mathcal{K}(X), \bar{f})$ partly imply the same for $(X, f)$. In 2014, Yang, Wang, and Li [13] studied the relationship between non-autonomous dynamical system and its hyperspace system in the aspect of sensitivity. In 2015, Wu, Wang, and Chen [14] had obtained a few sufficient and necessary conditions to ensure a dynamical system be $\mathcal{F}$-sensitive or multi-sensitive.

Inspired by the literature [13]-[17], this paper further studies some stronger forms of sensitivity in set-valued discrete systems. The structure of this paper is as follows. In Section 2, some basic definitions are given. In Section 3, the main results are established and proved.

\section{Preliminaries}

\subsection{Non-Autonomous Discrete System}

In this paper, let $X=[0,1]$, and the metric on $X$ is denoted as $\rho$. $f_{n}: X \rightarrow X, n \in \mathbb{N}$ is a mapping sequence, and denoted by $f_{1, \infty}=\left(f_{1}, f_{2}, \cdots\right)$. This sequence defines a non-autonomous discrete system $\left(X, f_{1, \infty}\right)$. Under this mapping sequence, the orbit of the point $x \in X$ is

$\operatorname{Orb}\left(x, f_{1, \infty}\right)=\left(f_{1}^{n}(x)\right)(n \in \mathbb{N})$, where $f_{1}^{n}=f_{n} \circ \cdots \circ f_{1}, f_{1}^{0}$ denotes the identity mapping. Similarly, $f_{n}^{k}=f_{n+k-1} \circ \cdots \circ f_{n+1} \circ f_{n}$.

\subsection{Set-Valued Product Systems}

Let $\mathcal{K}(X)$ be the hyperspace on $X$. That is, the space of nonempty compact subsets of $X$ with the Hausdorff metric $d_{H}$ defined by

$$
d_{H}(A, B)=\max \left\{\sup _{x \in A} \inf _{y \in B} d(x, y), \operatorname{supinf}_{y \in B} d(x, y)\right\}
$$

for any $A, B \in \mathcal{K}(X)$. Clearly, $\left(\mathcal{K}(X), d_{H}\right)$ is a compact metric space. The system $\left(X, f_{1, \infty}\right)$ induces a set-valued dynamical system $\left(\mathcal{K}(X), \bar{f}_{1, \infty}\right)$, where $\bar{f}_{1, \infty}: \mathcal{K}(X) \rightarrow \mathcal{K}(X)$ is defined as $\bar{f}_{1, \infty}(A)=f_{1, \infty}(A)$ for any $A \in \mathcal{K}(X)$. For any finite collection $A_{1}, \cdots, A_{n}$ of nonempty subsets of $X$, let

$$
\left\langle A_{1}, \cdots, A_{n}\right\rangle=\left\{A \in \mathcal{K}(X): A \subset \bigcup_{i=1}^{n} A_{i}, A \cap A_{i} \neq \varnothing, 1 \leq i \leq n\right\},
$$

where the topology on $\mathcal{K}(X)$ given by the metric $d_{H}$ is the same as the Vietoris or finite topology, which is generated by a basis consisting of all sets of the following form: $\left\langle U_{1}, \cdots, U_{n}\right\rangle$, where $\left\{U_{1}, \cdots, U_{n}\right\}$ is an arbitrary finite collection of nonempty open subsets of $X$.

Two set-valued non-autonomous discrete systems $\bar{f}_{1, \infty}: \mathcal{K}(X) \rightarrow \mathcal{K}(X)$ and $\bar{g}_{1, \infty}: \mathcal{K}(Y) \rightarrow \mathcal{K}(Y)$ are defined in compact metric spaces $\mathcal{K}(X)$ and $\mathcal{K}(Y)$, whose metrics are $d_{H_{1}}$ and $d_{H_{2}}$. Let $\overline{f_{1, \infty} \times g_{1, \infty}}: \mathcal{K}(X \times Y) \rightarrow \mathcal{K}(X \times Y)$, define $\overline{f_{1, \infty} \times g_{1, \infty}}(A, B)=\left(\bar{f}_{1, \infty}(A), \bar{g}_{1, \infty}(B)\right)$, where $(A, B) \in \mathcal{K}(X \times Y)$. The metric of $\mathcal{K}(X \times Y)$ is $d_{H}$, and define $d_{H}\left(\left(A_{1}, B_{1}\right),\left(A_{2}, B_{2}\right)\right)=\max \left(d_{H_{1}}\left(A_{1}, A_{2}\right), d_{H_{2}}\left(B_{1}, B_{2}\right)\right)$, where $\left(A_{1}, B_{1}\right),\left(A_{2}, B_{2}\right) \in \mathcal{K}(X \times Y)$. Then the system $\left(\mathcal{K}(X \times Y), \overline{f_{1, \infty} \times g_{1, \infty}}\right)$ is called the product dynamical system of the two set-valued non-autonomous discrete 
systems.

\subsection{Definitions of Sensitivity}

In this section, some definitions of the sensitivity in set-valued discrete systems will be given.

Definition 1. ([14]) Let $\mathcal{P}$ be the collection of all subsets of $\mathbb{Z}^{+}$. A collection $\mathcal{F} \subset \mathcal{P}$ is called a Furstenberg family if it is hereditary upwards, i.e., $F_{1} \subset F_{2}$ and $F_{1} \subset \mathcal{F}$ imply $F_{2} \subset \mathcal{F}$. A family $\mathcal{F}$ is proper if it is a proper subset of $\mathcal{P}$, i.e. neither empty nor the whole $\mathcal{P}$.

Definition 2. ([15]) Let $X$ be a nonempty set and $F$ be a family of nonempty sets composed of subsets of $X$, if

1) $U, V \in F$ then $U \cap V \in F$;2) $U \in F$ and $U \subset V$ then $V \in F$, then $F$ is said to be a filterdual on the set $X$.

Definition 3. ([9]) Given $A \subset \mathbb{Z}^{+}$, its upper and lower densities are defined by

$$
\begin{aligned}
& \overline{\operatorname{Dens}}(A)=\limsup _{n \rightarrow+\infty} \frac{1}{n}|A \cap\{0, \cdots, n-1\}|, \\
& \underline{\operatorname{Dens}}(A)=\liminf _{n \rightarrow+\infty} \frac{1}{n}|A \bigcap\{0, \cdots, n-1\}| .
\end{aligned}
$$

If $\overline{\operatorname{Dens}}(A)=\underline{\operatorname{Dens}}(A)=\xi$, then the set $A$ has density $\operatorname{Dens}(A)=\xi$. Let $\bar{M}\left(0^{+}\right)=\{F \in \mathcal{B}: \overline{\operatorname{Dens}}(F)>0\}$, where $\mathcal{B}$ is the collection of all infinite subsets of $\mathbb{Z}^{+}$, then $\bar{M}\left(0^{+}\right)$is a Furstenberg family. It can be verified that

$$
\begin{aligned}
k \bar{M}\left(0^{+}\right) & =\left\{F \in \mathcal{P}: \mathbb{Z}^{+} \backslash F \notin \bar{M}\left(0^{+}\right)\right\} \\
& =\left\{F \in \mathcal{P}: \overline{\text { Dens }}\left(\mathbb{Z}^{+} \backslash F\right)=0\right\} \\
& =\{F \in \mathcal{P}: \operatorname{Dens}(F)=1\} .
\end{aligned}
$$

This implies that $k \bar{M}\left(0^{+}\right)$is a filterdual.

Definition 4. ([18] [19] [20] [21] [22]) Let $\left(\mathcal{K}(X), \bar{f}_{1, \infty}\right)$ be a system and let $\mathcal{F}$ be a Furstenberg family.

1) $\left(\mathcal{K}(X), \bar{f}_{1, \infty}\right)$ is sensitive, if there exists an $\delta>0$ such that for any $A \in \mathcal{K}(X)$ and any $\varepsilon>0$, there exist $B \in \mathcal{K}(X)$ with $d_{H}(A, B)<\varepsilon$ such that $d_{H}\left(\bar{f}_{1}^{n}(A), \bar{f}_{1}^{n}(B)\right)>\delta(n \in \mathbb{N})$;

2) $\left(\mathcal{K}(X), \bar{f}_{1, \infty}\right)$ is infinitely sensitive, if there exists an $\delta>0$ such that for any $A \in \mathcal{K}(X)$ and any $\varepsilon>0$, there exist $B \in \mathcal{K}(X)$ with $d_{H}(A, B)<\varepsilon$ such that $\limsup d_{H}\left(\bar{f}_{1}^{n}(A), \bar{f}_{1}^{n}(B)\right) \geq \delta(n \in \mathbb{N})$;

3) $\left(\mathcal{K}(X), f_{1, \infty}^{n \rightarrow+\infty}\right)$ is $\mathcal{F}$-sensitive, if there exists an $\delta>0$ such that for any $A \in \mathcal{K}(X)$ and any $\varepsilon>0$, there exist $B \in \mathcal{K}(X)$ with $d_{H}(A, B)<\varepsilon$ such that $\left\{n \in \mathbb{Z}^{+}: d_{H}\left(\bar{f}_{1}^{n}(A), \bar{f}_{1}^{n}(B)\right)>\delta\right\} \in \mathcal{F}$;

4) $\left(\mathcal{K}(X), \bar{f}_{1, \infty}\right)$ is $\left(\mathcal{F}_{1}, \mathcal{F}_{2}\right)$-sensitive with the sensitive constant $\lambda$ if for any $A \in \mathcal{K}(X)$ and any $\varepsilon>0$, there exist $B \in \mathcal{K}(X)$ with $d_{H}(A, B)<\varepsilon$ such that $\left\{n \in \mathbb{Z}^{+}: d_{H}\left(\bar{f}_{1}^{n}(A), \bar{f}_{1}^{n}(B)\right)>\delta\right\} \in \mathcal{F}_{2}$ and $\left\{n \in \mathbb{Z}^{+}: d_{H}\left(\bar{f}_{1}^{n}(A), \bar{f}_{1}^{n}(B)\right)<\delta\right\} \in \mathcal{F}_{1}$ for any $\delta>0$; 
5) $\left(\mathcal{K}(X), \bar{f}_{1, \infty}\right)$ is ergodically sensitive if there exists $\varepsilon>0$ (ergodically sensitive constant) such that for any nonempty open subset $U \subset \mathcal{K}(X)$, $\left\{n \in \mathbb{Z}^{+}: \operatorname{diam}\left(\bar{f}_{1}^{n}(U)\right)>\varepsilon\right\} \in \bar{M}\left(0^{+}\right)$. Where $\operatorname{diam}(\cdot)$ is the diagonal of the set.

\section{Main Results}

Based on the definitions in Section 2, we now further investigate the dynamical properties of the product systems.

Lemma 1. ([14]) Let $X, Y$ be two compact metrics. Then, for any $A \in \mathcal{K}(X \times Y)$ and any $\varepsilon>0$, there exist nonempty open subsets $U_{1}, U_{2}, \cdots, U_{n} \subset X$ and $V_{1}, V_{2}, \cdots, V_{n} \subset Y$ such that $A \in\left\langle U_{1} \times V_{1}, \cdots, U_{n} \times V_{n}\right\rangle \subset\left\langle\overline{U_{1} \times V_{1}}, \cdots, \overline{U_{n} \times V_{n}}\right\rangle \subset B_{d_{H}}(A, \varepsilon)$.

Theorem 1. Let $\left(\mathcal{K}(X), \bar{f}_{1, \infty}\right)$ and $\left(\mathcal{K}(Y), \bar{g}_{1, \infty}\right)$ be two set-valued nonautonomous discrete systems, then $\left(\mathcal{K}(X \times Y), \frac{f_{1, \infty} \times g_{1, \infty}}{}\right)$ is sensitive if and only if $\left(\mathcal{K}(X), \bar{f}_{1, \infty}\right)$ or $\left(\mathcal{K}(Y), \bar{g}_{1, \infty}\right)$ is sensitive.

Proof. (Necessity) Assuming that $\left(\mathcal{K}(X), \bar{f}_{1, \infty}\right)$ and $\left(\mathcal{K}(Y), \bar{g}_{1, \infty}\right)$ are not sensitive, it is proved that $\left(\mathcal{K}(X \times Y), \overline{f_{1, \infty} \times g_{1, \infty}}\right)$ is not sensitive.

1) If $\left(\mathcal{K}(X), \bar{f}_{1, \infty}\right)$ is not sensitive, then for any $\delta_{1}>0$, there exist $A_{1} \in \mathcal{K}(X)$ and $\varepsilon>0$, there is $d_{H_{1}}\left(A_{1}, B_{1}\right) \geq \varepsilon$ for any $B_{1} \in \mathcal{K}(X)$ satisfying that

$$
d_{H_{1}}\left(\bar{f}_{1}^{n}\left(A_{1}\right), \bar{f}_{1}^{n}\left(B_{1}\right)\right) \leq \delta_{1} .
$$

2) If $\left(\mathcal{K}(Y), \bar{g}_{1, \infty}\right)$ is not sensitive, then for any $\delta_{2}>0$, there exist $A_{2} \in \mathcal{K}(Y)$ and $\varepsilon>0$, there is $d_{H_{2}}\left(A_{2}, B_{2}\right) \geq \varepsilon$ for any $B_{2} \in \mathcal{K}(Y)$ satisfying that

$$
d_{H_{2}}\left(\bar{g}_{1}^{n}\left(A_{2}\right), \bar{g}_{1}^{n}\left(B_{2}\right)\right) \leq \delta_{2} .
$$

Since $A_{1} \in \mathcal{K}(X), A_{2} \in \mathcal{K}(Y)$, then there is $A_{1} \times A_{2} \in \mathcal{K}(X \times Y)$ for any $B_{1} \times B_{2} \in \mathcal{K}(X \times Y)$, one has that

$$
d_{H}\left(A_{1} \times A_{2}, B_{1} \times B_{2}\right)=\max \left\{d_{H_{1}}\left(A_{1}, B_{1}\right), d_{H_{2}}\left(A_{2}, B_{2}\right)\right\} \geq \varepsilon .
$$

So

$$
\begin{aligned}
& d_{H}\left(\overline{f_{1}^{n} \times g_{1}^{n}}\left(A_{1}, A_{2}\right), \overline{f_{1}^{n} \times g_{1}^{n}}\left(B_{1}, B_{2}\right)\right) \\
& =d_{H}\left(\left(\bar{f}_{1}^{n}\left(A_{1}\right), \bar{g}_{1}^{n}\left(A_{2}\right)\right),\left(\bar{f}_{1}^{n}\left(B_{1}\right), \bar{g}_{1}^{n}\left(B_{2}\right)\right)\right) \\
& =\max \left\{d_{H_{1}}\left(\bar{f}_{1}^{n}\left(A_{1}\right), \bar{f}_{1}^{n}\left(B_{1}\right)\right), d_{H_{2}}\left(\bar{g}_{1}^{n}\left(A_{2}\right), \bar{g}_{1}^{n}\left(B_{2}\right)\right)\right\} .
\end{aligned}
$$

Let $\delta=\max \left(\delta_{1}, \delta_{2}\right)$, then

$$
d_{H}\left(\overline{f_{1}^{n} \times g_{1}^{n}}\left(A_{1}, A_{2}\right), \overline{f_{1}^{n} \times g_{1}^{n}}\left(B_{1}, B_{2}\right)\right) \leq \delta .
$$

Therefore, $\left(\mathcal{K}(X \times Y), \overline{f_{1, \infty} \times g_{1, \infty}}\right)$ is not sensitive and contradicts the proposition. So $\left(\mathcal{K}(X), \bar{f}_{1, \infty}\right)$ or $\left(\mathcal{K}(Y), \bar{g}_{1, \infty}\right)$ is sensitive.

(Sufficiency) For any nonempty open set $V \in \mathcal{K}(X \times Y)$, by Lemma 1, there exist nonempty open subsets $U_{1}, U_{2}, \cdots, U_{n} \subset X$ and $V_{1}, V_{2}, \cdots, V_{n} \subset Y$ such that 


$$
\left\langle\overline{U_{1} \times U_{n}}, \cdots, \overline{V_{1} \times V_{n}}\right\rangle \subset V
$$

If $\left(\mathcal{K}(X), \bar{f}_{1, \infty}\right)$ is sensitive, then there exists $\delta>0$, for any $A \in\left\langle\overline{U_{1}}, \cdots, \overline{U_{n}}\right\rangle$ and $\varepsilon>0$, there is $B \in\left\langle\overline{U_{1}}, \cdots, \overline{U_{n}}\right\rangle$ such that $d_{H_{1}}(A, B)<\varepsilon$, one has that

$$
d_{H_{1}}\left(\bar{f}_{1}^{n}(A), \bar{f}_{1}^{n}(B)\right)>\delta \text {. }
$$

Take any point $v_{i}$ in $V_{i}$, where $i \in\{1,2, \cdots, n\}$. Define $A_{i}=A \cap \overline{U_{i}}$, $B_{i}=B \cap \overline{U_{i}}, \quad \tilde{A}_{i}=A_{i} \times\left\{v_{i}\right\}, \tilde{B}_{i}=B_{i} \times\left\{v_{i}\right\}$. Therefore,

$$
\begin{aligned}
& A=\bigcup_{i=1}^{n} A_{i}, B=\bigcup_{i=1}^{n} B_{i}, \tilde{A}=\bigcup_{i=1}^{n} \tilde{A}_{i} \in\left\langle\overline{U_{1} \times V_{1}}, \cdots, \overline{U_{n} \times V_{n}}\right\rangle, \\
& \tilde{B}=\bigcup_{i=1}^{n} \tilde{B}_{i} \in\left\langle\overline{U_{1} \times V_{1}}, \cdots, \overline{U_{n} \times V_{n}}\right\rangle .
\end{aligned}
$$

Then for any $\tilde{A} \in \mathcal{K}(X \times Y)$, there exists $\tilde{B} \in \mathcal{K}(X \times Y)$ such that

$$
d_{H}(\tilde{A}, \tilde{B})=d_{H}\left(\bigcup_{i=1}^{n}\left(A_{i} \times\left\{v_{i}\right\}\right), \bigcup_{i=1}^{n}\left(B_{i} \times\left\{v_{i}\right\}\right)\right)<\varepsilon
$$

So

$$
\begin{aligned}
& d_{H}\left(\overline{f_{1}^{n} \times g_{1}^{n}}(\tilde{A}), \overline{f_{1}^{n} \times g_{1}^{n}}(\tilde{B})\right) \\
& =d_{H}\left(\bigcup_{i=1}^{n} \bar{f}_{1}^{n}\left(A_{i}\right) \times \bar{g}_{1}^{n}\left(\left\{v_{i}\right\}\right), \bigcup_{i=1}^{n} \bar{f}_{1}^{n}\left(B_{i}\right) \times \bar{g}_{1}^{n}\left(\left\{v_{i}\right\}\right)\right) \\
& \geq d_{H_{1}}\left(\bar{f}_{1}^{n}(A), \bar{f}_{1}^{n}(B)\right) \\
& >\delta .
\end{aligned}
$$

Hence, $\left(\mathcal{K}(X \times Y), \overline{f_{1, \infty} \times g_{1, \infty}}\right)$ is sensitive.

Theorem 2. Let $\left(\mathcal{K}(X), \bar{f}_{1, \infty}\right)$ and $\left(\mathcal{K}(Y), \bar{g}_{1, \infty}\right)$ be two set-valued nonautonomous discrete systems, then $\left(\mathcal{K}(X \times Y), \overline{f_{1, \infty} \times g_{1, \infty}}\right)$ is infinitely sensitive if and only if $\left(\mathcal{K}(X), \bar{f}_{1, \infty}\right)$ or $\left(\mathcal{K}(Y), \bar{g}_{1, \infty}\right)$ is infinitely sensitive.

Proof. (Necessity) Assuming that $\left(\mathcal{K}(X), \bar{f}_{1, \infty}\right)$ and $\left(\mathcal{K}(Y), \bar{g}_{1, \infty}\right)$ are not infinitely sensitive, it is proved that $\left(\mathcal{K}(X \times Y), \overline{f_{1, \infty} \times g_{1, \infty}}\right)$ is not infinitely sensitive.

1) If $\left(\mathcal{K}(X), \bar{f}_{1, \infty}\right)$ is not infinitely sensitive, then for any $\delta_{1}>0$, there exist $A_{1} \in \mathcal{K}(X)$ and $\varepsilon>0$, there is $d_{H_{1}}\left(A_{1}, B_{1}\right) \geq \varepsilon$ for any $B_{1} \in \mathcal{K}(X)$ satisfying that

$$
\limsup _{n \rightarrow+\infty} d_{H_{1}}\left(\bar{f}_{1}^{n}\left(A_{1}\right), \bar{f}_{1}^{n}\left(B_{1}\right)\right)<\delta_{1} .
$$

2) If $\left(\mathcal{K}(Y), \bar{g}_{1, \infty}\right)$ is not infinitely sensitive, then for any $\delta_{2}>0$, there exist $A_{2} \in \mathcal{K}(Y)$ and $\varepsilon>0$, there is $d_{H_{2}}\left(A_{2}, B_{2}\right) \geq \varepsilon$ for any $B_{2} \in \mathcal{K}(Y)$ satisfying that

$$
\limsup _{n \rightarrow+\infty} d_{H_{2}}\left(\bar{g}_{1}^{n}\left(A_{2}\right), \bar{g}_{1}^{n}\left(B_{2}\right)\right)<\delta_{2} .
$$

Since $A_{1} \in \mathcal{K}(X), A_{2} \in \mathcal{K}(Y)$, then there is $A_{1} \times A_{2} \in \mathcal{K}(X \times Y)$ for any $B_{1} \times B_{2} \in \mathcal{K}(X \times Y)$, one has that

$$
d_{H}\left(A_{1} \times A_{2}, B_{1} \times B_{2}\right)=\max \left\{d_{H_{1}}\left(A_{1}, B_{1}\right), d_{H_{2}}\left(A_{2}, B_{2}\right)\right\} \geq \varepsilon .
$$


So

$$
\begin{aligned}
& d_{H}\left(\overline{f_{1}^{n} \times g_{1}^{n}}\left(A_{1}, A_{2}\right), \overline{f_{1}^{n} \times g_{1}^{n}}\left(B_{1}, B_{2}\right)\right) \\
& =d_{H}\left(\left(\bar{f}_{1}^{n}\left(A_{1}\right), \bar{g}_{1}^{n}\left(A_{2}\right)\right),\left(\bar{f}_{1}^{n}\left(B_{1}\right), \bar{g}_{1}^{n}\left(B_{2}\right)\right)\right) \\
& =\max \left\{d_{H_{1}}\left(\bar{f}_{1}^{n}\left(A_{1}\right), \bar{f}_{1}^{n}\left(B_{1}\right)\right), d_{H_{2}}\left(\bar{g}_{1}^{n}\left(A_{2}\right), \bar{g}_{1}^{n}\left(B_{2}\right)\right)\right\} .
\end{aligned}
$$

Let $\delta=\max \left(\delta_{1}, \delta_{2}\right)$, then

$$
\begin{aligned}
& \limsup _{n \rightarrow+\infty} d_{H}\left(\overline{f_{1}^{n} \times g_{1}^{n}}\left(A_{1}, A_{2}\right), \overline{f_{1}^{n} \times g_{1}^{n}}\left(B_{1}, B_{2}\right)\right) \\
& =\max \left\{\limsup _{n \rightarrow+\infty} d_{H_{1}}\left(\bar{f}_{1}^{n}\left(A_{1}\right), \bar{f}_{1}^{n}\left(B_{1}\right)\right), \limsup _{n \rightarrow+\infty} d_{H_{2}}\left(\bar{g}_{1}^{n}\left(A_{2}\right), \bar{g}_{1}^{n}\left(B_{2}\right)\right)\right\} \\
& <\delta .
\end{aligned}
$$

Therefore, $\left(\mathcal{K}(X \times Y), \overline{f_{1, \infty} \times g_{1, \infty}}\right)$ is not infinitely sensitive and contradicts the proposition. So $\left(\mathcal{K}(X), \bar{f}_{1, \infty}\right)$ or $\left(\mathcal{K}(Y), \bar{g}_{1, \infty}\right)$ is infinitely sensitive.

(Sufficiency) For any nonempty open set $V \in \mathcal{K}(X \times Y)$, we know from Lemma 1, there exist nonempty open subsets $U_{1}, U_{2}, \cdots, U_{n} \subset X$ and $V_{1}, V_{2}, \cdots, V_{n} \subset Y$ such that

$$
\left\langle\overline{U_{1} \times U_{n}}, \cdots, \overline{V_{1} \times V_{n}}\right\rangle \subset V
$$

If $\left(\mathcal{K}(X), \bar{f}_{1, \infty}\right)$ is infinitely sensitive, then there exists $\delta>0$, for any $A \in\left\langle\overline{U_{1}}, \cdots, \overline{U_{n}}\right\rangle$ and $\varepsilon>0$, there is $B \in\left\langle\overline{U_{1}}, \cdots, \overline{U_{n}}\right\rangle$ such that $d_{H}(A, B)<\varepsilon$, one has that

$$
\limsup _{n \rightarrow+\infty} d_{H_{1}}\left(\bar{f}_{1}^{n}(A), \bar{f}_{1}^{n}(B)\right) \geq \delta .
$$

Define $\tilde{A}, \tilde{B}$ are the same as Theorem 3.1, then

$$
d_{H}(\tilde{A}, \tilde{B})=d_{H}\left(\bigcup_{i=1}^{n}\left(A_{i} \times\left\{v_{i}\right\}\right), \bigcup_{i=1}^{n}\left(B_{i} \times\left\{v_{i}\right\}\right)\right)<\varepsilon
$$

So

$$
\begin{aligned}
& \limsup _{n \rightarrow+\infty} d_{H}\left(\overline{f_{1}^{n} \times g_{1}^{n}}(\tilde{A}), \overline{f_{1}^{n} \times g_{1}^{n}}(\tilde{B})\right) \\
& =\limsup _{n \rightarrow+\infty} d_{H}\left(\bigcup_{i=1}^{n} \bar{f}_{1}^{n}\left(A_{i}\right) \times \bar{g}_{1}^{n}\left(\left\{v_{i}\right\}\right), \bigcup_{i=1}^{n} \bar{f}_{1}^{n}\left(B_{i}\right) \times \bar{g}_{1}^{n}\left(\left\{v_{i}\right\}\right)\right) \\
& \geq \limsup _{n \rightarrow+\infty} d_{H_{1}}\left(\bar{f}_{1}^{n}(A), \bar{f}_{1}^{n}(B)\right) \\
& \geq \delta .
\end{aligned}
$$

Therefore, $\left(\mathcal{K}(X \times Y), \overline{f_{1, \infty} \times g_{1, \infty}}\right)$ is infinitely sensitive.

Theorem 3. Let $\left(\mathcal{K}(X), \bar{f}_{1, \infty}\right)$ and $\left(\mathcal{K}(Y), \bar{g}_{1, \infty}\right)$ be two set-valued nonautonomous discrete systems, then $\left(\mathcal{K}(X \times Y), \frac{f_{1, \infty} \times g_{1, \infty}}{}\right)$ is $\mathcal{F}$-sensitive if and only if $\left(\mathcal{K}(X), \bar{f}_{1, \infty}\right)$ or $\left(\mathcal{K}(Y), \bar{g}_{1, \infty}\right)$ is $\mathcal{F}$-sensitive.

Proof. (Necessity) Assuming that $\left(\mathcal{K}(X), \bar{f}_{1, \infty}\right)$ and $\left(\mathcal{K}(Y), \bar{g}_{1, \infty}\right)$ are not $\mathcal{F}$-sensitive, it is proved that $\left(\mathcal{K}(X \times Y), \overline{f_{1, \infty} \times g_{1, \infty}}\right)$ is not $\mathcal{F}$-sensitive.

1) If $\left(\mathcal{K}(X), \bar{f}_{1, \infty}\right)$ is not $\mathcal{F}$-sensitive, then for any $\delta_{1}>0$, there exist $A_{1} \in \mathcal{K}(X)$ and $\varepsilon>0$, there is $d_{H_{1}}\left(A_{1}, B_{1}\right) \geq \varepsilon$ for any $B_{1} \in \mathcal{K}(X)$ satisfy- 
ing that

$$
\left\{n \in \mathbb{Z}^{+}: d_{H_{1}}\left(\bar{f}_{1}^{n}\left(A_{1}\right), \bar{f}_{1}^{n}\left(B_{1}\right)\right)>\delta_{1}\right\} \notin \mathcal{F} .
$$

2) If $\left(\mathcal{K}(Y), \bar{g}_{1, \infty}\right)$ is not $\mathcal{F}$-sensitive, then for any $\delta_{2}>0$, there exist $A_{2} \in \mathcal{K}(Y)$ and $\varepsilon>0$, there is $d_{H_{2}}\left(A_{2}, B_{2}\right) \geq \varepsilon$ for any $B_{2} \in \mathcal{K}(Y)$ satisfying that

$$
\left\{n \in \mathbb{Z}^{+}: d_{H_{2}}\left(\bar{g}_{1}^{n}\left(A_{2}\right), \bar{g}_{1}^{n}\left(B_{2}\right)\right)>\delta_{2}\right\} \notin \mathcal{F} .
$$

Since $A_{1} \in \mathcal{K}(X), A_{2} \in \mathcal{K}(Y)$, then there is $A_{1} \times A_{2} \in \mathcal{K}(X \times Y)$ for any $B_{1} \times B_{2} \in \mathcal{K}(X \times Y)$, one has that

$$
d_{H}\left(A_{1} \times A_{2}, B_{1} \times B_{2}\right)=\max \left\{d_{H_{1}}\left(A_{1}, B_{1}\right), d_{H_{2}}\left(A_{2}, B_{2}\right)\right\} \geq \varepsilon .
$$

So

$$
\begin{aligned}
& d_{H}\left(\overline{f_{1}^{n} \times g_{1}^{n}}\left(A_{1}, A_{2}\right), \overline{f_{1}^{n} \times g_{1}^{n}}\left(B_{1}, B_{2}\right)\right) \\
& =d_{H}\left(\left(\bar{f}_{1}^{n}\left(A_{1}\right), \bar{g}_{1}^{n}\left(A_{2}\right)\right),\left(\bar{f}_{1}^{n}\left(B_{1}\right), \bar{g}_{1}^{n}\left(B_{2}\right)\right)\right) \\
& =\max \left\{d_{H_{1}}\left(\bar{f}_{1}^{n}\left(A_{1}\right), \bar{f}_{1}^{n}\left(B_{1}\right)\right), d_{H_{2}}\left(\bar{g}_{1}^{n}\left(A_{2}\right), \bar{g}_{1}^{n}\left(B_{2}\right)\right)\right\} .
\end{aligned}
$$

Let $\delta=\max \left(\delta_{1}, \delta_{2}\right)$, then

$$
\left\{n \in \mathbb{Z}^{+}: d_{H}\left(\overline{f_{1}^{n} \times g_{1}^{n}}\left(A_{1}, A_{2}\right), \overline{f_{1}^{n} \times g_{1}^{n}}\left(B_{1}, B_{2}\right)\right)>\delta\right\} \notin \mathcal{F} .
$$

Therefore, $\left(\mathcal{K}(X \times Y), \overline{f_{1, \infty} \times g_{1, \infty}}\right)$ is not $\mathcal{F}$-sensitive and contradicts the proposition. So $\left(\mathcal{K}(X), \bar{f}_{1, \infty}\right)$ or $\left(\mathcal{K}(Y), \bar{g}_{1, \infty}\right)$ is $\mathcal{F}$-sensitive.

(Sufficiency) For any nonempty open set $V \in \mathcal{K}(X \times Y)$, we know from Lemma 1 , there exist nonempty open subsets $U_{1}, U_{2}, \cdots, U_{n} \subset X$ and $V_{1}, V_{2}, \cdots, V_{n} \subset Y$ such that

$$
\left\langle\overline{U_{1} \times U_{n}}, \cdots, \overline{V_{1} \times V_{n}}\right\rangle \subset V
$$

If $\left(\mathcal{K}(X), \bar{f}_{1, \infty}\right)$ is $\mathcal{F}$-sensitive, then there exists $\delta>0$, for any $A \in\left\langle\overline{U_{1}}, \cdots, \overline{U_{n}}\right\rangle$ and $\varepsilon>0$, there is $B \in\left\langle\overline{U_{1}}, \cdots, \overline{U_{n}}\right\rangle$ such that $d_{H_{1}}(A, B)<\varepsilon$, one has that

$$
\left\{n \in \mathbb{Z}^{+}: d_{H_{1}}\left(\bar{f}_{1}^{n}(A), \bar{f}_{1}^{n}(B)\right)>\delta\right\} \in \mathcal{F} .
$$

By the same proof as Theorem 3.1, one can obtain that

$$
d_{H}\left(\overline{f_{1}^{n} \times g_{1}^{n}}(\tilde{A}), \overline{f_{1}^{n} \times g_{1}^{n}}(\tilde{B})\right) \geq d_{H_{1}}\left(\bar{f}_{1}^{n}(A), \bar{f}_{1}^{n}(B)\right) .
$$

Combining this with the hereditary upwards property of $\mathcal{F}$, it follows that

$$
\begin{aligned}
& \left\{n \in \mathbb{Z}^{+}: d_{H_{1}}\left(\bar{f}_{1}^{n}(A), \bar{f}_{1}^{n}(B)\right)>\delta\right\} \\
& \subset\left\{n \in \mathbb{Z}^{+}: d_{H}\left(\overline{f_{1}^{n} \times g_{1}^{n}}(\tilde{A}), \overline{f_{1}^{n} \times g_{1}^{n}}(\tilde{B})\right)>\delta\right\} \in \mathcal{F} .
\end{aligned}
$$

Hence, $\left(\mathcal{K}(X \times Y), \overline{f_{1, \infty} \times g_{1, \infty}}\right)$ is $\mathcal{F}$-sensitive.

Theorem 4. Let $\left(\mathcal{K}(X), \bar{f}_{1, \infty}\right)$ and $\left(\mathcal{K}(Y), \bar{g}_{1, \infty}\right)$ be two set-valued nonautonomous discrete systems, if $\left(\mathcal{K}(X \times Y), \overline{f_{1, \infty} \times g_{1, \infty}}\right)$ is $\left(\mathcal{F}_{1}, \mathcal{F}_{2}\right)$-sensitive 
then $\left(\mathcal{K}(X), \bar{f}_{1, \infty}\right)$ or $\left(\mathcal{K}(Y), \bar{g}_{1, \infty}\right)$ is $\left(\mathcal{F}_{1}, \mathcal{F}_{2}\right)$-sensitive.

Proof. Assuming that $\left(\mathcal{K}(X), \bar{f}_{1, \infty}\right)$ and $\left(\mathcal{K}(Y), \bar{g}_{1, \infty}\right)$ are not $\left(\mathcal{F}_{1}, \mathcal{F}_{2}\right)$ sensitive, it is proved that $\left(\mathcal{K}(X \times Y), \overline{f_{1, \infty} \times g_{1, \infty}}\right)$ is not $\left(\mathcal{F}_{1}, \mathcal{F}_{2}\right)$-sensitive.

1) If $\left(\mathcal{K}(X), \bar{f}_{1, \infty}\right)$ is not $\left(\mathcal{F}_{1}, \mathcal{F}_{2}\right)$-sensitive, then there is $\delta_{1}>0$, there exist $A_{1} \in \mathcal{K}(X)$ and $\varepsilon>0$, there is $d_{H_{1}}\left(A_{1}, B_{1}\right) \geq \varepsilon$ for any $B_{1} \in \mathcal{K}(X)$ satisfying that

$$
\begin{aligned}
& \left\{n \in \mathbb{Z}^{+}: d_{H_{1}}\left(\bar{f}_{1}^{n}\left(A_{1}\right), \bar{f}_{1}^{n}\left(B_{1}\right)\right)>\delta_{1}\right\} \notin \mathcal{F}_{2}, \\
& \left\{n \in \mathbb{Z}^{+}: d_{H_{1}}\left(\bar{f}_{1}^{n}\left(A_{1}\right), \bar{f}_{1}^{n}\left(B_{1}\right)\right)<\delta_{1}\right\} \notin \mathcal{F}_{1} .
\end{aligned}
$$

2) If $\left(\mathcal{K}(Y), \bar{g}_{1, \infty}\right)$ is not $\left(\mathcal{F}_{1}, \mathcal{F}_{2}\right)$-sensitive, then there is $\delta_{2}>0$, there exist $A_{2} \in \mathcal{K}(Y)$ and $\varepsilon>0$, there is $d_{H_{2}}\left(A_{2}, B_{2}\right) \geq \varepsilon$ for any $B_{2} \in \mathcal{K}(Y)$ satisfying that

$$
\begin{aligned}
& \left\{n \in \mathbb{Z}^{+}: d_{H_{2}}\left(\bar{g}_{1}^{n}\left(A_{2}\right), \bar{g}_{1}^{n}\left(B_{2}\right)\right)>\delta_{2}\right\} \notin \mathcal{F}_{2}, \\
& \left\{n \in \mathbb{Z}^{+}: d_{H_{2}}\left(\bar{g}_{1}^{n}\left(A_{2}\right), \bar{g}_{1}^{n}\left(B_{2}\right)\right)<\delta_{2}\right\} \notin \mathcal{F}_{1} .
\end{aligned}
$$

Since $A_{1} \in \mathcal{K}(X), A_{2} \in \mathcal{K}(Y)$, then there is $A_{1} \times A_{2} \in \mathcal{K}(X \times Y)$ for any $B_{1} \times B_{2} \in \mathcal{K}(X \times Y)$, one has that

$$
d_{H}\left(A_{1} \times A_{2}, B_{1} \times B_{2}\right)=\max \left\{d_{H_{1}}\left(A_{1}, B_{1}\right), d_{H_{2}}\left(A_{2}, B_{2}\right)\right\} \geq \varepsilon .
$$

So

$$
\begin{aligned}
& d_{H}\left(\overline{f_{1}^{n} \times g_{1}^{n}}\left(A_{1}, A_{2}\right), \overline{f_{1}^{n} \times g_{1}^{n}}\left(B_{1}, B_{2}\right)\right) \\
& =d_{H}\left(\left(\bar{f}_{1}^{n}\left(A_{1}\right), \bar{g}_{1}^{n}\left(A_{2}\right)\right),\left(\bar{f}_{1}^{n}\left(B_{1}\right), \bar{g}_{1}^{n}\left(B_{2}\right)\right)\right) \\
& =\max \left\{d_{H_{1}}\left(\bar{f}_{1}^{n}\left(A_{1}\right), \bar{f}_{1}^{n}\left(B_{1}\right)\right), d_{H_{2}}\left(\bar{g}_{1}^{n}\left(A_{2}\right), \bar{g}_{1}^{n}\left(B_{2}\right)\right)\right\} .
\end{aligned}
$$

Let $\delta=\max \left(\delta_{1}, \delta_{2}\right)$, then

$$
\begin{aligned}
& \left\{n \in \mathbb{Z}^{+}: d_{H}\left(\overline{f_{1}^{n} \times g_{1}^{n}}\left(A_{1}, A_{2}\right), \overline{f_{1}^{n} \times g_{1}^{n}}\left(B_{1}, B_{2}\right)\right)>\delta\right\} \notin \mathcal{F}_{2}, \\
& \left\{n \in \mathbb{Z}^{+}: d_{H}\left(\overline{f_{1}^{n} \times g_{1}^{n}}\left(A_{1}, A_{2}\right), \overline{f_{1}^{n} \times g_{1}^{n}}\left(B_{1}, B_{2}\right)\right)<\delta\right\} \notin \mathcal{F}_{1} .
\end{aligned}
$$

Therefore, $\left(\mathcal{K}(X \times Y), \overline{f_{1, \infty} \times g_{1, \infty}}\right)$ is not $\left(\mathcal{F}_{1}, \mathcal{F}_{2}\right)$-sensitive and contradicts the proposition. So $\left(\mathcal{K}(X), \bar{f}_{1, \infty}\right)$ or $\left(\mathcal{K}(Y), \bar{g}_{1, \infty}\right)$ is $\left(\mathcal{F}_{1}, \mathcal{F}_{2}\right)$-sensitive.

Lemma 2. ([14]) Let $\left(X, f_{1, \infty}\right)$ be a non-autonomous dynamical system, for any nonemptyopen set $U \subset X$ and any $n \in \mathbb{Z}^{+}$, one has $\operatorname{diam} f_{1}^{n}(U)=\operatorname{diam} \bar{f}_{1}^{n}(\langle U\rangle)$.

Theorem 5. Let $\left(X, f_{1, \infty}\right)$ and $\left(Y, g_{1, \infty}\right)$ be two non-autonomous dynamical systems and let $\bar{M}\left(0^{+}\right)$be a Furstenberg family such that $k \bar{M}\left(0^{+}\right)$is a filterdual. If $\left(\mathcal{K}(X \times Y), \overline{f_{1, \infty} \times g_{1, \infty}}\right)$ is ergodically sensitive, then $\left(X, f_{1, \infty}\right)$ or $\left(Y, g_{1, \infty}\right)$ is ergodically sensitive.

Proof. Suppose that both $f_{1, \infty}$ and $g_{1, \infty}$ are not ergodically sensitive. Then, for any $\varepsilon>0$, there exist nonempty open subsets $U \subset X$ and $V \subset Y$ such that 


$$
\left\{n \in \mathbb{Z}^{+}: \operatorname{diam}\left(f_{1}^{n}(U)\right)>\varepsilon / 2\right\} \notin \bar{M}\left(0^{+}\right),
$$

and

$$
\left\{n \in \mathbb{Z}^{+}: \operatorname{diam}\left(g_{1}^{n}(V)\right)>\varepsilon / 2\right\} \notin \bar{M}\left(0^{+}\right) .
$$

So

$$
\begin{aligned}
F_{1} & :=\left\{n \in \mathbb{Z}^{+}: \operatorname{diam}\left(f_{1}^{n}(U)\right) \leq \varepsilon / 2\right\} \\
& =\mathbb{Z}^{+} \backslash\left\{n \in \mathbb{Z}^{+}: \operatorname{diam}\left(f_{1}^{n}(U)\right)>\varepsilon / 2\right\} \in k \bar{M}\left(0^{+}\right),
\end{aligned}
$$

and

$$
\begin{aligned}
F_{2} & :=\left\{n \in \mathbb{Z}^{+}: \operatorname{diam}\left(g_{1}^{n}(V)\right) \leq \varepsilon / 2\right\} \\
& =\mathbb{Z}^{+} \backslash\left\{n \in \mathbb{Z}^{+}: \operatorname{diam}\left(g_{1}^{n}(V)\right)>\varepsilon / 2\right\} \in k \bar{M}\left(0^{+}\right) .
\end{aligned}
$$

Since $k \bar{M}\left(0^{+}\right)$is a filterdual, then $F_{1} \cap F_{2} \in k \bar{M}\left(0^{+}\right)$. For any $n \in F_{1} \cap F_{2}$, noting that $\operatorname{diam}_{1}^{n}(U) \leq \varepsilon / 2$ and $\operatorname{diam}_{1}^{n}(V) \leq \varepsilon / 2$, applying Lemma 3.2, one has that

$$
\begin{aligned}
\operatorname{diam}\left(\overline{f_{1}^{n} \times g_{1}^{n}}(\langle U \times V\rangle)\right) & =\operatorname{diam}\left(f_{1}^{n} \times g_{1}^{n}(U \times V)\right) \\
& =\operatorname{diam}\left(f_{1}^{n}(U) \times g_{1}^{n}(V)\right) \\
& \leq \sqrt{(\varepsilon / 2)^{2}+(\varepsilon / 2)^{2}} \\
& <\varepsilon .
\end{aligned}
$$

So

$$
F_{1} \cap F_{2} \subset\left\{n \in \mathbb{Z}^{+}: \operatorname{diam}\left(\overline{f_{1}^{n} \times g_{1}^{n}}(\langle U \times V\rangle)\right)<\varepsilon\right\} \in k \bar{M}\left(0^{+}\right) .
$$

This implies that

$$
\left\{n \in \mathbb{Z}^{+}: \operatorname{diam}\left(\overline{f_{1}^{n} \times g_{1}^{n}}(\langle U \times V\rangle)\right) \geq \varepsilon\right\} \notin \bar{M}\left(0^{+}\right) .
$$

Since $\varepsilon$ is arbitrary, it shows that $\overline{f_{1, \infty} \times g_{1, \infty}}$ is not ergodically sensitive. Therefore, $\left(X, f_{1, \infty}\right)$ or $\left(Y, g_{1, \infty}\right)$ is ergodically sensitive.

\section{Conclusion}

For set-valued non-autonomous discrete dynamical systems, the sensitivity of the product systems and the factor systems are consistent most of the time, for example, sensitive, infinitely sensitive, $\mathcal{F}$-sensitive, and $\left(\mathcal{F}_{1}, \mathcal{F}_{2}\right)$-sensitive, while it is not a necessary and sufficient condition for ergodically sensitive. Based on this paper and others, one can further consider some other chaotic properties of set-valued discrete systems, retention conditions for compound operation, and so on, which are worthy of study.

\section{Acknowledgements}

This work was funded by the Opening Project of Key Laboratory of Higher Education of Sichuan Province for Enterprise Informationalization and Internet 
of Things (No. 2020WZJ01), the Scientific Research Project of Sichuan University of Science and Engineering (No. 2020RC24), and the Graduate student Innovation Fund (Nos. y2020077, y2021100)

\section{Conflicts of Interest}

The authors declare no conflicts of interest regarding the publication of this paper.

\section{References}

[1] Román-Flores, H. (2003) A Note on Transitivity in Set-Valued Discrete Systems. Chaos, Solitons and Fractals, 17, 99-104. https://doi.org/10.1016/S0960-0779(02)00406-X

[2] Sánchez, I., Sanchis, M. and Villanueva, H. (2017) Chaos in Hyperspaces of Nonautonomous Discrete Systems. Chaos, Solitons and Fractals: The Interdisciplinary Journal of Nonlinear Science, and Nonequilibrium and Complex Phenomena, 94, 68-74. https://doi.org/10.1016/j.chaos.2016.11.009

[3] Peris, A. (2005) Set-Valued Discrete Chaos. Chaos, Solitons and Fractals, 26, 19-23. https://doi.org/10.1016/j.chaos.2004.12.039

[4] Gu, R.B. and Guo, W.J. (2006) On Mixing Property in Set-Valued Discrete Systems. Chaos, Solitons and Fractals, 28, 747-754. https://doi.org/10.1016/j.chaos.2005.04.004

[5] Liu, L. and Peng, D.M. (2017) A Note on Transitive Points of Set-Valued Discrete Systems. Advances in Fixed Point Theory, 7, 214-220.

[6] Román-Flores, H. and Chalco-Cano, Y. (2005) Robinson's Chaos in Set-Valued Discrete Systems. Chaos, Solitons and Fractals, 25, 33-42. https://doi.org/10.1016/j.chaos.2004.11.006

[7] Fedeli, A. (2005) On Chaotic Set-Valued Discrete Dynamical Systems. Chaos, Solitons and Fractals, 23, 1381-1384. https://doi.org/10.1016/S0960-0779(04)00394-7

[8] Gu, R.B. (2007) Kato's Chaos in Set-Valued Discrete Systems. Chaos, Solitons and Fractals, 31, 765-771. https://doi.org/10.1016/j.chaos.2005.10.041

[9] Shao, H. and Zhu, H. (2019) Chaos in Non-Autonomous Discrete Systems and Their Induced Set-Valued Systems. Chaos, 29, Article ID: 033117. https://doi.org/10.1063/1.5054867

[10] Liu, H., Shi, E.H. and Liao, G.F. (2009) Sensitivity of Set-Valued Discrete Systems. Nonlinear Analysis, 71, 6122-6125. https://doi.org/10.1016/j.na.2009.06.003

[11] Liu, H., Lei, F.C., Wang, L.D. and Gao, H.Y. (2013) Li-Yorke Sensitivity of Set-Valued Discrete Systems. Journal of Applied Mathematics, 2013, Article ID: 260856. https://doi.org/10.1155/2013/260856

[12] Sharma, P. and Nagar, A. (2010) Inducing Sensitivity on Hyperspaces. Topology and Its Applications, 157, 2052-2058. https://doi.org/10.1016/j.topol.2010.05.002

[13] Yang, C.Y., Wang, Y.G. and Li, Z.M. (2014) Some Results about Sensitive on Non-Autonomous Dynamical System in Hyperspace. Pure and Applied Mathematics, 30, 201-206.

[14] Wu, X.X., Wang, J.J. and Chen, G.R. (2015) $\mathcal{F}$-Sensitivity and Multi-Sensitivity of Hyperspatial Dynamical Systems. Journal of Mathematical Analysis and Applications, 429, 16-26. https://doi.org/10.1016/j.jmaa.2015.04.009

[15] Wang, Y.G., Wei, G. and Campbell, W.H. (2009) Sensitive Dependence on Initial 
Conditions between Dynamical Systems and Their Induced Hyperspace Dynamical Systems. Topology and its Applications, 156, 803-811.

https://doi.org/10.1016/j.topol.2008.10.014

[16] Liao, G.F., Wang, L.D. and Zhang, Y.C. (2006) Transitivity, Mixing and Chaos for a Class of Set-Valued Mappings. Science in China Series A, 49, 1-8.

https://doi.org/10.1007/s11425-004-5234-5

[17] Wu, X.X. and Zhu, P.Y. (2012) Devaney Chaos and Li-Yorke Sensitivity for Product Systems. Akadémiai Kiadó, 49, 538-548.

https://doi.org/10.1556/sscmath.49.2012.4.1226

[18] Moothathu, T.K. (2007) Stronger Forms of Sensitivity for Dynamical Systems. Nonlinearity, 20, 2115-2126. https://doi.org/10.1088/0951-7715/20/9/006

[19] Wu, X.X. and Zhu, P.Y. (2013) Dense Chaos and Densely Chaotic Operators. Tsukuba Journal of Mathematics, 36, 367-375. https://doi.org/10.21099/tkbjm/1358777004

[20] Li, R.S., Zhao, Y., Wang, H.Q. and Liang, H.H. (2020) Stronger Forms of Transitivity and Sensitivity for Non-Autonomous Discrete Dynamical Systems and Furstenberg Families. Journal of Dynamical and Control Systems, 26, 109-126. https://doi.org/10.1007/s10883-019-09437-6

[21] Li, R.S. (2012) A Note on Stronger Forms of Sensitivity for Dynamical Systems. Chaos, Solitons and Fractals, 45, 753-758. https://doi.org/10.1016/j.chaos.2012.02.003

[22] Vasisht, R. and Das, R. (2018) On Stronger Forms of Sensitivity in Non-Autonomous Systems. Taiwanese Journal of Mathematics, 22, 1139-1159.

https://doi.org/10.11650/tjm/180406 